\title{
Direct CP Violation and Rare Decays at Belle
}

\author{
George W.S. Hou ${ }^{\mathrm{a}}$ \\ a Department of Physics, National Taiwan University, Taipei, Taiwan 10617
}

The highlights of direct $C P$ violation (DCPV) and hadronic rare decays by the Belle experiment are presented.

\section{MACHINE, DETECTOR, BASIC METHOD}

The Belle experiment operates at the asymmetric energy $e^{+} e^{-}$collider, KEK-B, on the $\Upsilon(4 S)$ resonance, with $\beta \gamma=0.425$. KEK-B has achieved a peak luminosity of $15.81 \mathrm{nb}^{-1} \mathrm{~s}^{-1}$, with $>1$ $\mathrm{fb}^{-1}$ per day, and, as of summer 2005, Belle has accumulated a total of $469.8 \mathrm{fb}^{-1}$ of data.

Besides tracking (SVD and CDC), EM calorimeter and also $K_{L} / \mu$ detection, one major element for the DCPV/Rare program is the Aerogel Cherenkov Counter, ACC, a threshold Cherenkov device with several $n$ values. Together with $d E / d x$ from CDC and TOF information, for $p_{K}>500 \mathrm{MeV}$, one has kaon efficiency of order $80 \%$, with pion fake rate of order $10 \%$ or less.

With precise knowledge of $E_{\text {beam }}$ in c.m.s., together with predominance of $\Upsilon(4 S) \rightarrow B \bar{B}$ decay, $B$ signal reconstruction utilizes the $M_{b c}$ and $\Delta E$ projections. A set of modified Fox-Wolfram moments is used to reject the continuum $q \bar{q}$ background via an $\mathcal{L R}$ likelihood ratio cut.

\section{OBSERVATION OF DCPV IN $K \pi$}

DCPV in $B$ decays, defined as

$\mathcal{A}_{C P}=\frac{\Gamma(\bar{B} \rightarrow \bar{f})-\Gamma(B \rightarrow f)}{\Gamma(\bar{B} \rightarrow \bar{f})+\Gamma(B \rightarrow f)}$,

was first attempted by CLEO in 1999. In 2004, Belle and BaBar both measured DCPV in $B^{0} \rightarrow$ $K^{+} \pi^{-}$, with combined significance $>5 \sigma$. With $386 \mathrm{M} B \bar{B}$ pairs, Belle [1] now finds $3026 \pm 63$ signal events, giving $\mathcal{A}_{C P}\left(K^{+} \pi^{-}\right)=-0.113 \pm$ 0.022 (stat.) \pm 0.008 (syst.) with $\sim 5 \sigma$ significance (see Fig. 1). The current world average is $-0.115 \pm 0.018[2]$, and DCPV in the $B$ system is firmly established. Together with evidence for DCPV in $\pi^{+} \pi^{-}$, i.e. $\mathcal{A}_{C P}\left(\pi^{+} \pi^{-}\right) \equiv \mathcal{A}_{\pi \pi} \equiv$ $-\mathcal{C}_{\pi \pi}=0.37 \pm 0.10$ [2] (caution: Belle and BaBar differ by $2.3 \sigma$ ), there is sizable strong phase between tree $(T)$ and penguin $(P)$ amplitudes.

For $B^{+} \rightarrow K^{+} \pi^{0}$ decay, the $386 \mathrm{M}$ update finds $1084 \pm 45$ signal events, giving [1] $\mathcal{A}_{C P}\left(K^{+} \pi^{0}\right)=$ $0.04 \pm 0.04 \pm 0.02$. This implies [2] $\mathcal{A}_{C P}\left(K^{+} \pi^{0}\right)-$ $\mathcal{A}_{C P}\left(K^{+} \pi^{-}\right) \neq 0$ at $>3.8 \sigma$ significance, and hints at a larger than expected color-suppressed $C$, or a large electroweak penguin $P_{E W}$.

\section{OBSERVATION OF $\pi^{0} \pi^{0}$}

A sizable $C$ is indicated by a larger than expected $B^{0} \rightarrow \pi^{0} \pi^{0}$ rate. The $B^{0} \rightarrow \pi^{0} \pi^{0}$ mode
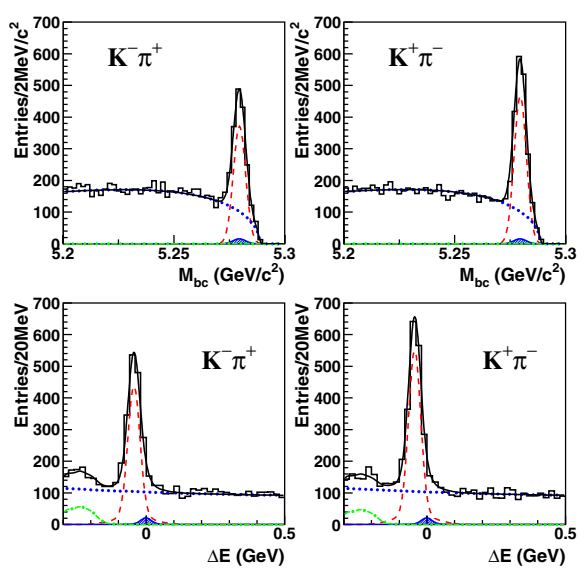

Figure 1. $M_{b c}$ and $\Delta E$ projections for $K^{-} \pi^{+}$vs. $K^{+} \pi^{-}$events with $386 \mathrm{M} B \bar{B}$. 


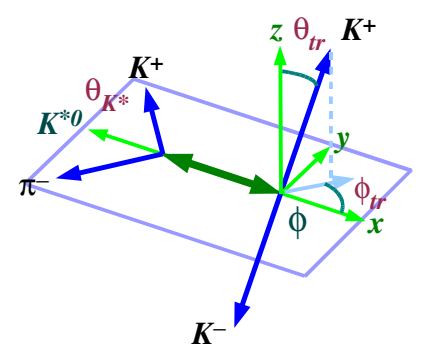

Figure 2. Definition of transversity basis.

has been observed by Belle with $275 \mathrm{M} B \bar{B}$, giving $\mathcal{B}\left(B^{0} \rightarrow \pi^{0} \pi^{0}\right)=\left(2.3_{-0.5-0.3}^{+0.4+0.2}\right) \times 10^{-6}$ with $5.8 \sigma$ significance. This confirms the 2003 evidence from BaBar and Belle, and the current combined result is $(1.45 \pm 0.29) \times 10^{-6}[2]$. The value is not fully understood, but clearly suggest that $C$ is not as suppressed as anticipated.

A first attempt was made on DCPV, using same flavor-tagging techniques as the timedependent CPV program. The result is [3] $\mathcal{A}_{C P}\left(\pi^{0} \pi^{0}\right)=0.44_{-0.52}^{+0.53} \pm 0.17$, and the combined world average is $0.28_{-0.39}^{+0.40}[2]$. While not yet significant, this is now the main limiting factor for $\phi_{2}$ extraction from the $\pi \pi$ program.

\section{POLARIZATION IN $\phi K^{*}, \rho^{+} K^{* 0}$}

For charmless $B \rightarrow V V$ decays, longitudinal polarization is expected to be dominant, with $1-f_{L}$ suppressed by $\mathcal{O}\left(m_{V}^{2} / m_{B}^{2}\right)$. This is true for most $T$ dominated decays measured to date, such as in $\rho \rho$ and $\rho \omega$ modes. However, for $B \rightarrow \phi K^{*}$, which is a pure penguin $b \rightarrow s \bar{s} s$ transition, it was found $[4,5]$ that $f_{L}\left(\phi K^{*}\right) \sim 0.5$ only. Theoretical suggestions to account for this range from annihilation, rescattering or transversity of emitted gluon, to new physics.

The first full helicity amplitude analysis was conducted by Belle [4], based on $85.5 \mathrm{M} B \bar{B}$. In part motivated by search for new physics, Belle has updated [6] with $275 \mathrm{M} B \bar{B}$ (with comparable results for $\phi K^{* 0}$ by BaBar [7], based on 227M $B \bar{B})$. Signal yields are extracted by fits to $\Delta E$, $M_{b c}$ and $M_{K K}$, with $f_{0}(980) K^{*}$ and $K \bar{K}$ (non$\phi) K^{*}$ components taken into account when making the fit. $173 \pm 16$ and $85_{-11}^{+12}$ signal events were
Table 1

Fitted amplitudes for $B \rightarrow \phi K^{*}$.

\begin{tabular}{lcc}
\hline \hline & $\phi K^{* 0}$ & $\phi K^{*+}$ \\
\hline$\left|A_{0}\right|^{2}$ & $0.45 \pm 0.05 \pm 0.02$ & $0.52 \pm 0.08 \pm 0.03$ \\
$\left|A_{\perp}\right|^{2}$ & $0.30 \pm 0.06 \pm 0.02$ & $0.19 \pm 0.08 \pm 0.02$ \\
$\arg A_{\|}$ & $2.39 \pm 0.24 \pm 0.04$ & $2.10 \pm 0.28 \pm 0.04$ \\
$\arg A_{\perp}$ & $2.51 \pm 0.23 \pm 0.04$ & $2.31 \pm 0.30 \pm 0.07$ \\
\hline \hline
\end{tabular}

extracted for neutral and charged $B$, respectively. No indication for DCPV was found.

To study $\phi K^{*}$ polarization, the transversity basis (see Fig. 2) is used. The $z$ direction is out of $K^{*}$ decay plane, the $\phi$ direction defines the $x$ axis, with $y$ axis on same side of kaon from $K^{*}$ decay. Then $\theta_{K^{*}}$ is the angle between the $K^{*}$ direction and its daughter kaon direction in the $K^{*}$ rest frame, and $\theta_{t r}, \phi_{t r}$ are the polar and azimuthal angles, in $\phi$ rest frame, of $K^{+}$from $\phi$ decay.

Denoting the longitudinal amplitude as $A_{0}$, $A_{\perp}$ and $A_{\|}$are the transverse polarized components along $z$ and $y$ axis. Normalizing to $\left|A_{0}\right|^{2}+\left|A_{\perp}\right|^{2}+\left|A_{\|}\right|^{2}=1$ and taking $A_{0}$ as real, we make an unbinned maximum likelihood fit to $\phi K^{*}$ candidates in the signal region. The fitted parameters $\left|A_{0}\right|^{2},\left|A_{\perp}\right|^{2}$ and $\arg A_{\perp}, \arg A_{\|}$are shown in Table 1. The results for $\phi K^{* 0}$ and $\phi K^{*+}$ are consistent with each other, and $f_{L}=\left|A_{0}\right|^{2} \simeq 0.5$ is confirmed. From $\arg A_{\perp}$, $\arg A_{\|}$, we see $4.3 \sigma \mathrm{ev-}$ idence for final state interactions (FSI, or strong phase), i.e. away from $\pi$.

The $T$-odd quantities

$A_{T}^{0}=\operatorname{Im}\left(A_{\perp} A_{0}^{*}\right), \quad A_{T}^{\|}=\operatorname{Im}\left(A_{\perp} A_{\|}^{*}\right)$,

measure triple products of momentum and polarization vectors, and probe $T$-odd $C P$-violation. We find $A_{T}^{0}=0.13_{-0.14}^{+0.11} \pm 0.04$ vs. $\bar{A}_{T}^{0}=0.28 \pm$ $0.08 \pm 0.01$, and likewise $A_{T}^{\|}=0.03 \pm 0.08 \pm 0.01$ vs. $\bar{A}_{T}^{\|}=0.03 \pm 0.06 \pm 0.01$, showing no significant difference between $B$ and $\bar{B}$ decays.

A more sophisticated angular analysis has been performed on $B^{0}$ vs. $\bar{B}^{0}$ decays. It confirms the longitudinal fraction $f_{L}$ and $C P$-odd fraction $f_{\perp}$, as well as the nonzero effect that arises from FSI. But variables that should vanish in absence of new physics are all consistent with zero.

Belle has studied [8] polarization in another 


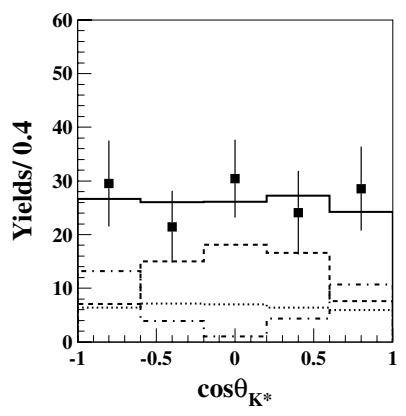

Figure 3. Fit to $\rho^{+} K^{* 0}$ helicity distributions. The dot-dash, dash and dotted histograms are the $A_{0}, A_{ \pm}$and non-resonant $\rho K \pi$, respectively.

pure penguin $V V$ decay, $B^{+} \rightarrow \rho^{+} K^{* 0}$, which is $b \rightarrow s \bar{d} d$. Out of $275 \mathrm{M} B \bar{B}$ pairs, $135 \pm 17$ $K^{+} \pi^{+} \pi^{-} \pi^{0}$ events are found, which includes nonresonant backgrounds. Selecting the nominal $K^{* 0}$ region, the $\pi^{+} \pi^{0}$ is predominantly $\rho$, hence there is little $K^{* 0} \pi^{+} \pi^{0}$ background. However, selecting the nominal $\rho$ region, besides a dominant $K^{* 0}$ component, one finds a significant non-resonant $\rho^{+} K^{+} \pi^{-}$component in $M\left(K^{+} \pi^{-}\right)$plot. This is taken into account in the angular analysis.

We perform a binned simultaneous $\chi^{2}$ fit to the $\rho$ and $K^{*}$ helicity-angle distributions. An $S$-wave $K \pi$ system is assumed in the modelling of nonresonant $\rho^{+} K^{+} \pi^{-}$component. The fit result is shown in Fig. 3 for the helicity angle $\cos \theta_{K^{*}}$. The fitted longitudinal fraction is $f_{L}\left(\rho^{+} K^{* 0}\right)=0.43 \pm$ $0.11_{-0.02}^{+0.05}$, which is consistent with $\phi K^{*}$ case, and far below the expected $f_{L} \sim 1$. The branching fraction is found to be $\mathcal{B}\left(\rho^{+} K^{* 0}\right)=(8.9 \pm 1.7 \pm$ $1.2) \times 10^{-6}$. Note, however, that some discrepancy exists with the BaBar result [9] based on $89 \mathrm{M}$ $B \bar{B}$, which finds $f_{L}=0.79 \pm 0.08 \pm 0.04 \pm 0.02$ and $\mathcal{B}=\left(17.0 \pm 2.9 \pm 2.0_{-1.9}^{+0.0}\right) \times 10^{-6}$, where the last error is from non-resonant background.

\section{Khh DALITZ ANALYSIS}

Belle pioneered the Dalitz analysis of threebody $K^{+} h^{+} h^{-}$decays, where $h=\pi, K$. A recent update [10] with $152 \mathrm{M} B \bar{B}$ yields $1533 \pm 69$ $K^{+} \pi^{+} \pi^{-}$events and $1089 \pm 41 K^{+} K^{+} K^{-}$events. A first amplitude analysis gives clear signals in $K^{* 0} \pi^{+}, f_{0}(980) K^{+}, \phi K^{+}$, and $\chi_{c 0} K^{+}$, includ-
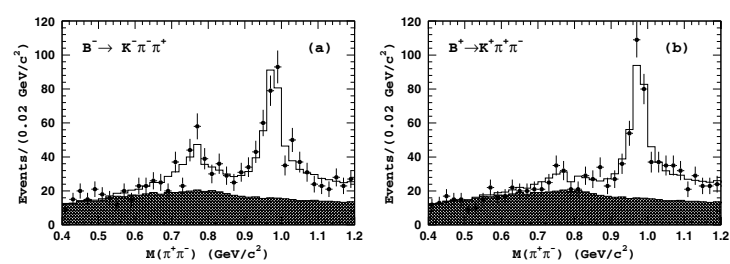

Figure 4. $M\left(\pi^{+} \pi^{-}\right)$spectra for (a) $K^{-} \pi^{-} \pi^{+}$and (b) $K^{+} \pi^{+} \pi^{-}$, without helicity cut.

ing the first observation of $\rho^{0} K^{+} . K_{0}^{*}(1430)^{0} \pi^{+}$ is observed, but rate determination suffers large model error. Broad structures are also seen in $M\left(\pi^{+} \pi^{-}\right)\left(M\left(K^{+} K^{-}\right)\right)$at $1300(1500) \mathrm{GeV} / \mathrm{c}^{2}$. Non-resonant $K^{+} \pi^{+} \pi^{-}$and $K^{+} K^{+} K^{-}$decays comprise a significant fraction of the total threebody signal, which presaged the $\rho^{+} K^{+} \pi^{-}$case.

Belle has recently updated [11] with $386 \mathrm{M}$ for $K^{ \pm} \pi^{ \pm} \pi^{\mp}$, with an aim towards search for DCPV. Simultaneous fit to $B^{-}$and $B^{+}$yields $2248 \pm 79 K^{-} \pi^{-} \pi^{+}$signal events vs. $2038 \pm$ 76 for $K^{+} \pi^{+} \pi^{-}$. The resulting asymmetry is $\mathcal{A}_{C P}\left(K^{+} \pi^{+} \pi^{-}\right)=0.049 \pm 0.026 \pm 0.030$, which is consistent with zero. However, amplitude analysis allows DCPV in various quasi-two-body channels to be studied. The only channel where the statistical significance of the CP asymmetry exceeds the $3 \sigma$ level is $\rho^{0} K^{+}$.

We find $\mathcal{A}_{C P}\left(\rho^{0} K^{+}\right)=0.30 \pm 0.11 \pm 0.03_{-0.04}^{+0.11}$, which has $3.9 \sigma$ statistical significance. This asymmetry is illustrated in Fig. 4 without any helicity cut. A helicity cut of $\cos \theta_{\text {hel }}^{\pi \pi}<0$ would make the asymmetry even more apparent. The measured $\mathcal{A}_{C P}$ is consistent with the earlier result based on $275 \mathrm{M}$ [12], and consistent with the BaBar result [13] of $0.34 \pm 0.13 \pm 0.06_{-0.20}^{+0.15}$ based on $232 \mathrm{M} B \bar{B}$ pairs. Modelling error is still large, but if confirmed with a larger data sample, this would be the first observation of $C P$ violation in the decay of a charged meson.

\section{BARYONIC MODES}

In the $K^{+} h^{+} h^{-}$study, taking $h=\pi, K$ was following CLEO convention. Another possibility is $h=p$. Belle observed $p \bar{p} K^{+}$in 2002 [14], the first charmless baryonic decay mode of $B$ mesons to be observed. Subsequently, a host of charmless bary- 

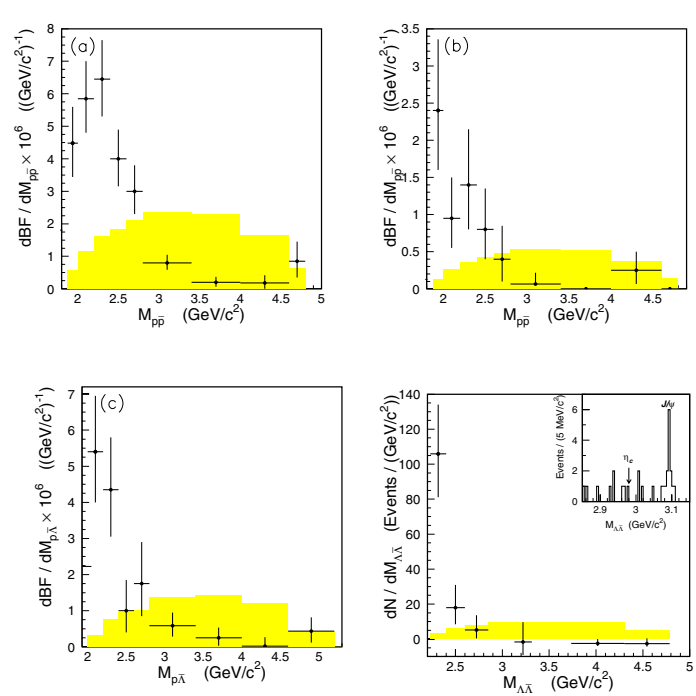

Figure 5. Baryon pair mass spectra for (a) $p \bar{p} K^{+}$, (b) $p \bar{p} K_{S}$, (c) $p \bar{\Lambda} \pi^{-}$and (d) $\Lambda \bar{\Lambda} K^{+}$.

onic modes have emerged: $p \bar{\Lambda} \pi^{+}, p \bar{p} \pi^{+}, p \bar{p} K^{0}$, $p \bar{p} K^{*+}, \Lambda \bar{\Lambda} K^{+}$, and also $p \bar{\Lambda} \gamma$. However, the corresponding two-body $p \bar{p}, \bar{\Lambda} p, \Lambda \bar{\Lambda}$ modes have not been seen [15], with $90 \%$ confidence level limits below $10^{-6}$, which is in contrast to the mesonic case. Another type of rare three-body baryonic decay, $J / \psi \bar{\Lambda} p$, has been observed by Belle [16] at the $10^{-5}$ level.

One common feature of the observed threebody charmless baryonic modes is "threshold peaking" in the low baryon-pair mass spectrum. A recent study with $152 \mathrm{M}$ gives $[17] \mathcal{B}\left(B^{+} \rightarrow\right.$ $\left.p \bar{p} K^{+}\right)=\left(5.30_{-0.39}^{+0.45} \pm 0.58\right) \times 10^{-6}, \mathcal{B}\left(B^{0} \rightarrow\right.$ $\left.p \bar{p} K_{S}\right)=\left(1.20_{-0.22}^{+0.32} \pm 0.14\right) \times 10^{-6}, \mathcal{B}\left(B^{0} \rightarrow\right.$ $\left.p \bar{\Lambda} \pi^{-}\right)=\left(3.27_{-0.51}^{+0.62} \pm 0.39\right) \times 10^{-6}$, confirming earlier results, and [18] the first observation of $\mathcal{B}\left(B^{+} \rightarrow \Lambda \bar{\Lambda} K^{+}\right)=\left(2.91_{-0.70}^{+0.90} \pm 0.38\right) \times 10^{-6}$. The corresponding baryon pair mass spectra are given in Fig. 5. Although the shape varies, all exhibit peaking behavior towards threshold, compared to phase space which is shaded. A similar study [19] with $152 \mathrm{M}$ finds $\mathcal{B}\left(B^{+} \rightarrow p \bar{\Lambda} \gamma\right)=$ $\left(2.16_{-0.53}^{+0.58} \pm 0.20\right) \times 10^{-6}$, and also exhibiting threshold peaking towards low $M(p \bar{\Lambda})$. This behavior is understood as resonant string fragmentation, while the three-body hadronic modes are not fully understood.
This work was supported by the National Science Council of Taiwan.

\section{REFERENCES}

1. K. Abe et al. [Belle Collaboration], hepex/0507045.

2. Heavy Flavor Averaging Group, see http://www.slac.stanford.edu/xorg/hfag/.

3. Y. Chao, P. Chang et al. [Belle Collaboration], Phys. Rev. Lett. 94, 181803 (2005).

4. K.-F. Chen, A. Bozek et al. [Belle Collaboration], Phys. Rev. Lett. 91, 201801 (2003).

5. B. Aubert et al. [BaBar Collaboration], Phys. Rev. Lett. 91, 171802 (2003).

6. K.-F. Chen et al. [Belle Collaboration], Phys. Rev. Lett. 94, 221804 (2005).

7. B. Aubert et al. [BaBar Collaboration], Phys. Rev. Lett. 93, 231804 (2004).

8. J. Zhang et al. [Belle Collaboration], Phys. Rev. Lett. 95, 141801 (2005).

9. B. Aubert et al. [BaBar Collaboration], hepex/0408093.

10. A. Garmash et al. [Belle Collaboration], Phys. Rev. D 71, 092003 (2005).

11. K. Abe et al. [Belle Collaboration], hepex/0509001.

12. A. Garmash et al. [Belle Collaboration], hepex/0505048.

13. B. Aubert et al. [BaBar Collaboration], hepex/0507004.

14. K. Abe et al. [Belle Collaboration], Phys. Rev. Lett. 88, 181803 (2002).

15. M.-C. Chang et al. [Belle Collaboration], Phys. Rev. D 71, 072007 (2005).

16. Q.L. Xie et al. [Belle Collaboration], hepex/0508011.

17. M.-Z. Wang et al. [Belle Collaboration], Phys. Lett. B 617, 141 (2005).

18. Y.-J. Lee, M.-Z. Wang et al. [Belle Collaboration], Phys. Rev. Lett. 93, 211801 (2004).

19. Y.-J. Lee, M.-Z. Wang et al. [Belle Collaboration], Phys. Rev. Lett. 95, 061802 (2005). 